\title{
Authors' reply: Disease prevention and sedentary lifestyle
}

\section{Olivier L. Charansonney and Jean-Pierre Després}

We would like to thank Brigid Lynch and her colleagues (Lynch, B. M., Healy, G. N., Dunstan, D. W. \& Owen, N. Sedentary versus inactive: distinctions for disease prevention. Nat. Rev. Cardiol. doi:10.1038/ nrcardio.2010.68-c1 $)^{1}$ for taking the time to comment on our Perspectives article (Charansonney, O. L. \& Després, J.-P. Disease prevention-should we target obesity or sedentary lifestyle? Nat. Rev. Cardiol. 7, 468-472 (2010)). ${ }^{2}$ In our model, we made the point that prolonged physical activity was an inherent and essential component of healthy human physiology and that lack of it would represent a stress that increases susceptibility to chronic metabolic diseases, especially in the context of exposure to excess calories. This Australian group has pioneered the important notion that a distinction had to be made between lack of physical activity and sedentary behavior. They have published evidence that clearly showed that, for any given level of physical activity, sedentary time (that is, watching television, or sitting in front of a computer for hours) had detrimental effects on cardiometabolic health..$^{3-5}$

We strongly support their view, which is fully consistent with our model. Globally, sedentary time is associated with accumulating stress signals that favor the pathological processes leading to chronic metabolic diseases. Accordingly, in order to evaluate individuals' risks of cardiometabolic diseases and premature death, both sedentary behaviors and level of physical activity should be assessed. We agree with Lynch and her colleagues that public health guidelines on physical activity fail to consider sedentary lifestyle. Therefore, meeting current physical activity guidelines does not necessarily prevent the deleterious metabolic consequences of sedentary lifestyle. Furthermore, these guidelines promote a minimal level of physical activity, which is far lower than the human physiological benchmark that we referred to in our paper. ${ }^{2}$

Finally, in the context of forced sedentary hours imposed by numerous professional occupations (including ours!), one question for which further work is warranted would be "Can robust physical activity or exercise attenuate the deleterious consequences of our sedentary work and, if so, how much physical activity or exercise would be required?". One thing is for sure: adding many hours of television watching, video games, or sitting in front of a computer during our leisure time will only exacerbate the consequences of our sedentary professional occupations.

Department of Cardiology, Centre Hospitalier Sud Francilien, 59 Boulevard Henri Dunant, 91108 Corbeil Essonnes Cedex, France

(O. L. Charansonney). Division of Kinesiology, Department of Social and Preventive Medicine, Université Laval, 2705 Boulevard Laurier, Québec, QC G1V 4G2, Canada (J.-P. Després).

Correspondence to: J.-P. Després jean-pierre.despres@criucpq.ulaval.ca

doi:10.1083/nrcardio.2010.68-c2

Competing interests

The authors declare no competing interests.

1. Lynch, B. M., Healy, G. N., Dunstan, D. W. $\&$ Owen, N. Sedentary versus inactive: distinctions for disease prevention. Nat. Rev. Cardiol. doi:10.1038/nrcardio.2010.68-c1.

2. Charansonney, O. L. \& Després, J. P. Disease prevention—should we target obesity or sedentary lifestyle? Nat. Rev. Cardiol. 7, 468-472 (2010).

3. Healy, G. N. et al. Television time and continuous metabolic risk in physically active adults. Med. Sci. Sports Exerc. 40, 639-645 (2008).

4. Thorp, A. A. et al. Deleterious associations of sitting time and television viewing time with cardiometabolic risk biomarkers-Australian Diabetes, Obesity and Lifestyle (AusDiab) study 2004-2005 Diabetes Care 33, 327-334 (2010).

5. Dunstan, D. W. et al. Television viewing time and mortality: The Australian Diabetes, Obesity and Lifestyle Study (AusDiab). Circulation 121, 384-391 (2010). 